\title{
Functionalization of graphite, glassy carbon, and polymer surfaces with highly oxidized sulfur species by plasma treatments
}

\author{
M. Collaud Coen a) \\ Department of Physics, University of Fribourg, Pérolles, CH-1700 Fribourg, Switzerland \\ B. Keller \\ Swiss Federal Laboratory for Materials Testing and Research, CH-8600 Dubendorf, Switzerland \\ P. Groening and L. Schlapbach ${ }^{\text {b) }}$ \\ Department of Physics, University of Fribourg, Pérolles, CH-1700 Fribourg, Switzerland
}

(Received 14 January 2002; accepted 30 July 2002)

\begin{abstract}
$\mathrm{SO}_{2}$ and $\mathrm{SO}_{2}+\mathrm{H}_{2} \mathrm{O}$ plasma treatments have been performed on highly oriented pyrolytic graphite, glassy carbon and several polymers in order to functionalize their surface with sulfur in different oxidation states. X-ray photoelectron spectroscopy and time-of-flight secondary ion mass spectroscopy measurements prove that highly oxidized sulfur species $\left(\mathrm{SO}_{3}, \mathrm{SO}_{3} \mathrm{H}, \mathrm{SO}_{4}\right.$, and $\mathrm{SO}_{4} \mathrm{H}$ ) as well as covalently bound sulfur and low oxidized sulphur species ( $\mathrm{SO}$ and $\mathrm{SO}_{2}$ ) are formed and chemisorbed at the surface by plasma treatment. The oxidation state of the adsorbed sulfur species depends on the bias potential applied to the sample. The greater the bias, the lower the oxidation state. The same behavior as a function of the plasma parameters is found for all substrates. The maximal coverage of the surface with sulfur functionalities has been estimated to be about one monolayer. The modifications of the surface topography have also been measured with atomic force microscopy. (C) 2002 American Institute of Physics. [DOI: 10.1063/1.1510170]
\end{abstract}

\section{INTRODUCTION}

The effect of plasma treatments on different kind of surfaces has been extensively studied during the last 20 years. Moreover, plasma treatments are now widely used in industry and are applied in various domains such as microelectronic, packaging, decorative and functional coatings, cleaning of all kind of materials, including sterilization of medical instruments. However, the effects and phenomena occurring during plasma treatments and inducing surface modifications are not yet completely understood. One of the ultimate goals of plasma treatments is to produce material with unmodified bulk properties and with particular and well-defined surface properties. This remains an important problem, particularly for the polymer industry dealing with adhesion and diffusion.

Surface pretreatments, and particularly plasma treatments, can induce various modifications on the polymer surfaces depending on the treatment conditions. Many studies have proven that substantial changes in the chemistry, electronic properties, wettability, and topography can be achieved by plasma treatments. ${ }^{1,2}$ However, these three kinds of modifications are most of the time closely correlated. To understand the causes of their appearance, these modifications have to be related to the internal plasma parameters, namely the plasma density, the electron temperature, the chemical activity of the ions and the bias potential that defines the kinetic energy of the ions hitting the sample. These internal parameters are defined by the external plasma parameters, namely the nature of the gas, the pressure, the po-

\footnotetext{
"Electronic mail: mattine,collaud@unifr.ch

b) Also at: Swiss Federal Laboratory for Materials Testing and Research, CH-8600 Dübendorf, Switzerland.
}

sition of the sample in the discharge zone, the rf bias applied to the sample, and the microwave power. By carefully controlling the treatment conditions, well-defined surface modifications, such as the incorporation of determined functional groups, can be produced.

This article describes chemical, physical, and topological modifications induced by $\mathrm{SO}_{2}$ plasma treatments on several polymers, graphite, and glassy carbon (GC). The incorporation of defined sulfur functionalities is of prime importance in a number of challenging research domains. The main goal to be achieved during this study was to chemisorb stable $\mathrm{SO}_{3} \mathrm{H}$ groups at the top surface in order to obtain a surface protonic conductivity. One direct application of protonic conductive surfaces is related to fuel cell technology. Platin is actually one essential component in the fuel cell that allows hydrogen and oxygen atoms to recombine and therefore to produce energy. Small clusters of platin can effectively be deposited instead of a full layer, if a protonic conductivity can be achieved between the clusters in order to activate them. The deposition of $\mathrm{SO}_{3} \mathrm{H}$ on glassy carbon $(\mathrm{GC})$ can therefore increase the efficiency and decrease the cost of fuel cells.

\section{EXPERIMENT}

The substrate materials for this study are highly oriented pyrolytic graphite (HOPG) from Advanced Ceramics Corporation, USA, GC Sigradur® from H.T.W., Germany, isotactic polypropylene (PP), polycarbonate (PC), and polyhydroxybutyrate-biopolymer (PHB) from Goodfellow, UK, and polyestherpolyuretane (TPU) from Sarnatech Xiro, $\mathrm{CH}$. 
The samples are treated in a low pressure plasma chamber, which has a base pressure of $5 \times 10^{-6} \mathrm{~Pa}$. The plasma is ignited by an electron cyclotron resonance process with permanent magnets at $2.45 \mathrm{GHz}$ microwave frequency. The sample can be capacitively coupled to a $13.56 \mathrm{MHz}$ if potential, so that a negative dc potential $\left(V_{\mathrm{dc}}\right)$ develops on the surface. The samples were treated with $\mathrm{SO}_{2}$. sometimes with additional gases $\left(\mathrm{H}_{2} \mathrm{O}, \mathrm{N}_{2}, \mathrm{O}_{2}\right)$ at pressures between 0.04 and $0.5 \mathrm{~Pa}$. The floating potential on the sample $\left(V_{f}\right)$ has always been measured between 0 and $-4 \mathrm{~V}$. The ion kinetic energy is then given by $E_{\text {kin }}=e^{*}\left(V_{p}-V_{f}\right)$, where $V_{p}$ is the plasma potential. The maximum dc potential depends on the adjustable if power and on the pressure; it is of the order of $-200 \mathrm{~V}$ for a dc power of $18 \mathrm{~W}$. Details of the plasma chamber have been discussed elsewhere. ${ }^{3}$ The plasma chamber is directly connected through a vacuum line to the $\mathrm{x}$-ray photoelectron spectrometer (XPS).

As surface analytical tools we used XPS, atomic force microscopy (AFM) and time-of-flight secondary ion mass spectroscopy (TOF-SMMS). XPS was performed in a VG ESCALAB 5 spectrometer. The analyses were carried out with nonmonochromatized $\mathrm{Mg} \mathrm{K} \alpha$ radiation ( $h \nu=1253.6$ $\mathrm{eV})$. High-resolution spectra were taken with $20 \mathrm{eV}$ pass energy, in the constant analyzer mode. The mean-free path of inelastic scattering for $1 \mathrm{keV}$ electrons in polymers is about 3 $\mathrm{nm}$, which results in an XPS probing depth of $10 \mathrm{~nm}$ for $\mathrm{Mg}$ $\mathrm{K} \alpha$ radiation. Element concentrations were evaluated from peak areas after Shirley background subtraction using theoretical cross section. Charging of the polymer samples during XPS, due to the photoelectron emission, was corrected by setting the binding energy of the main peak (hydrocarbon component) of the $\mathrm{C}$ 1s spectrum at $285.0 \mathrm{eV}$.

The modifications of the substrate topography induced by plasma treatments were investigated with an AFM (Nanoscope III, Digital Instruments, Santa Barbara) in the TappingMode ${ }^{\mathrm{TM}}$. The samples were scanned with a standard Si cantilever supplied by the microscope manufacturer. The measurements were done in air. The applied force, was always minimized, not to deform the samples. The roughness corresponds to the root-mean square of the height and was always calculated for a $5 \mu \mathrm{m} \times 5 \mu \mathrm{m}$ area.

TOF-SIMS spectra were recorded using a Physical Electronics 7200 system, equipped with an $8 \mathrm{keV} \mathrm{Cs}^{+}$primary ion gun and a reflectron-type mass analyzer. Under standard operation conditions, the instrument produced 1 ns full width at half maximum primary ion pulses, yielding a mass resolution $M / \Delta M>7000$ at $m / z=28$. The band pass energy of the reflectron was set to $70 \mathrm{eV}$. During data acquisition, the ion dose was kept in the range of $10^{11}$ ions $/ \mathrm{cm}^{2}$, well below the static secondary ion mass spectroscopy limit of $10^{13}$ ions $/ \mathrm{cm}^{2}$. Surface charging of insulating material was compensated with a low-energy electron flood gun.

\section{RESULTS AND DISCUSSION}

\section{A. Functionalization of highly oriented pyrolytic graphite, glassy carbon, and polypropylene surfaces with sulfur species of various oxidation degrees}

$\mathrm{SO}_{2}$ plasma treatments, sometimes with an adjunction of $\mathrm{H}_{2} \mathrm{O}$ gas, allow incorporating sulfur species with several de-

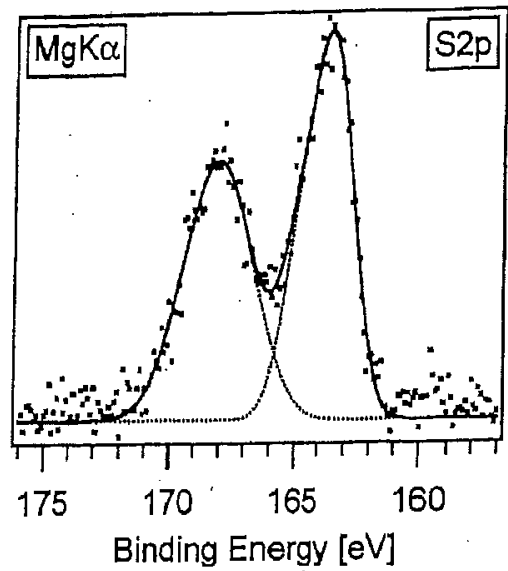

FIG. 1. XPS measured $S$ 2p spectra of $H O P G$ treated for 5 min in a $\mathrm{SO}_{2}$ $+\mathrm{H}_{2} \mathrm{O}$ plasma atmosphere. The low and highly oxidized sulfur peaks have been fitted with a double Gaussian peak corresponding to $S 2 p_{I / 2}$ and $\mathrm{S}_{2} p_{3 / 2}$.

grees of oxidation on various substrates. The amount and the chemical state of the sulfur atoms depend on the treatment conditions (see Sec. III C). Two chemical states of the S $2 p$ are clearly favored, as can be seen on the XPS spectra (Fig. 1). Both chemical species of the $S 2 p$ peaks have been fitted with double Gaussian curves, which take into account the $\mathrm{S} 2 p_{1 / 2}$ and $\mathrm{S} 2 p_{3 / 2}$ compounds. The first $\mathrm{S} 2 p_{1 / 2}$ peak has a binding energy of $164.0 \pm 0.4 \mathrm{eV}$ depending on the substrate and on the treatment. This component corresponds to a low oxidized sulfur species (SO), even if the sulfide component [binding energy $(\mathrm{BE})=163.7 \mathrm{eV}]^{4}$. is perhaps also sometimes present. The highly oxidized species is found at a $\mathrm{BE}$ of $168.5 \pm 0.5 \mathrm{eV}$, which is clearly higher than the $\mathrm{BE}$ of $\mathrm{SO}_{2}$ in polyethersulphone (PES) $(\mathrm{BE}=167.6 \mathrm{eV}){ }^{5}$ Siegbahn et al. ${ }^{6}$ have found a linear dependence between the measured XPS BE of the sulfur species and the corresponding charge of the sulfur atom. Table I gives the so-calculated charges on the sulfur atom for the different sulfur chemical states. The sulfur is therefore in a more oxidized state than in PES (O $=\mathrm{S}=\mathrm{O}$ ), namely in the $\mathrm{SO}_{3}(\mathrm{H})$ or $\mathrm{SO}_{4}(\mathrm{H})$ configuration.

Apart from sulfur, carbon and oxygen are naturally found in the treated surfaces. On all of the samples containing highly oxidized sulfur, nitrogen is also measured. Figure 2 shows the correlation between the oxygen and nitrogen content and the sulfur incorporated in an HOPG surface for different treatment conditions leading all to the incorporation of highly oxidized sulfur species. The $\mathrm{O} / \mathrm{S}$ ratio is always

TABLE I. BE of $S 2 p$ photoelectrons as a function of the charge on the sulfur atom.

\begin{tabular}{cc}
\hline XPS S $2 p$ BE $(\mathrm{eV})$ & Charge on S atoms $(q)$ \\
\hline 163.6 & 0.25 \\
164.0 & 0.30 \\
167.6 & 1.05 \\
168.0 & 1.10 \\
168.5 & 1.18 \\
169.0 & 1.30 \\
170.0 & 1.50 \\
\hline
\end{tabular}




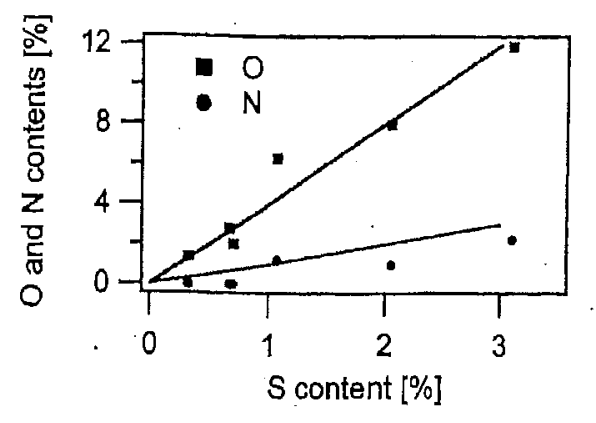

FIG. 2. Oxygen and nitrogen contents of $\mathrm{SO}_{2}+\mathrm{H}_{2} \mathrm{O}$ plasma treated $\mathrm{HOPG}$ surface at $1 \times 10^{-2}$ mbar for several pressures and treatment times.

about 4 and the N/S ratio about 1 , as it can be estimated from the two drawn lines with slopes equal to 1 and 4 , respectively, in Fig. 2. These proportions are found for all substrates that do not natively contain oxygen or nitrogen. This corroborates the aforementioned assessment saying that $\mathrm{SO}_{3}(\mathrm{H})$ or $\mathrm{SO}_{4}(\mathrm{H})$ functionalities are incorporated by plasma treatments.

The presence of nitrogen is not an artifact in the sense that it is found only when highly oxidized sulfur is formed. For treatment conditions similar but with higher ion energy, sulfur is incorporated as low oxidized species and no nitrogen is detected on the surface. In the first experiments, the HOPG samples have been pasted with silver glue. No nitrogen has been incorporated on these samples, but silver was found in a comparable ratio $(\mathrm{Ag} / \mathrm{S}=1)$. It seems, therefore, that the presence of nitrogen or of silver is necessary to the formation of $\mathrm{SO}_{4} \mathrm{H}$ during $\mathrm{SO}_{2}+\mathrm{H}_{2} \mathrm{O}$ plasma treatments. A tentatively explanation is to consider that the big $\mathrm{SO}_{4} \mathrm{H}$ groups need to be stabilized by a hydrogen bond with a primary amine group (Fig. 3). The primary amine is actually a very frequent substitute in a number of organic compounds. Very few nitrogen atoms are necessary to stabilize the $\mathrm{SO}_{4} \mathrm{H}$ groups, and both functionalities are found after long treatment times $(2-15 \mathrm{~min})$. Therefore, the nitrogen atoms originate probably as residual impurities, since the base pressure of the plasma chamber is of $10^{-6} \mathrm{mbar}$.

TOF-SIMS measurements have also been performed on HOPG and PP samples. Figure 4 shows that a great amount of highly oxidized sulfur species $\mathrm{SO}_{3}, \mathrm{SO}_{3} \mathrm{H}, \mathrm{SO}_{4}$, and $\mathrm{SO}_{4} \mathrm{H}$ are found on both surfaces. If the formation of $\mathrm{SO}_{3}$ and $\mathrm{SO}_{3} \mathrm{H}$ is favored on $\mathrm{HOPG}, \mathrm{SO}_{4} \mathrm{H}$ are preferentially found on PP. These different affinities between the substrates can be related to the greater facility to oxidize PP than HOPG. $\mathrm{CH}_{2} \mathrm{~N}$ functionalities at $m / e=28.019$ are also

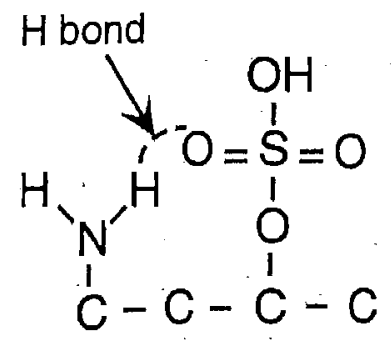

FIG. 3. Schematic view of the stabilizing function of nitrogen on highly oxidized sulfur.
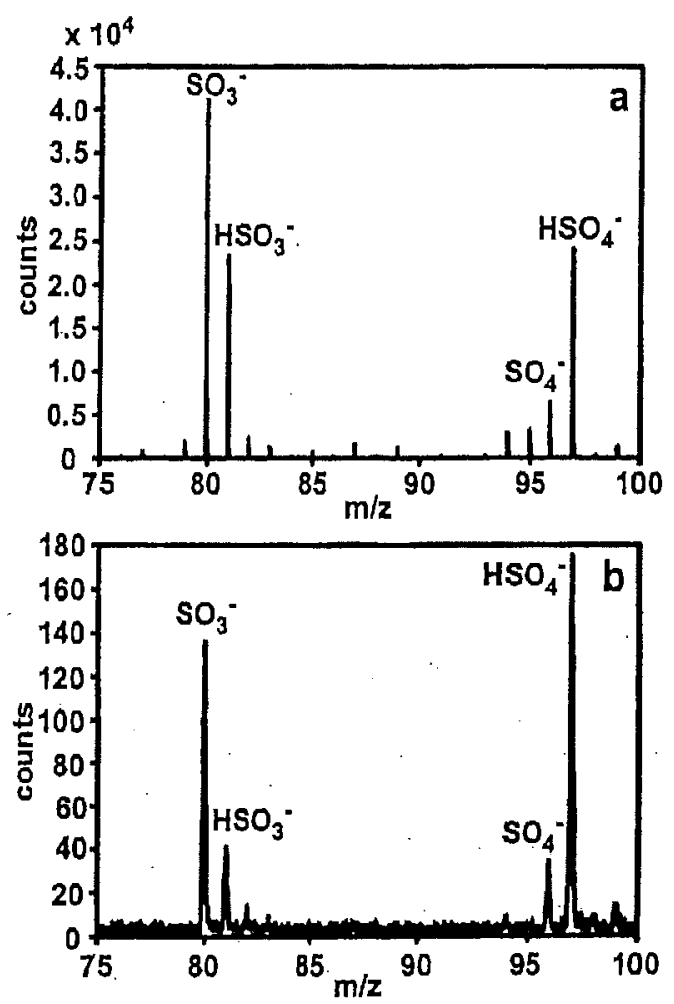

FIG. 4. TOF-SIMS spectra of (a) HOPG and (b) PP treated by $\mathrm{SO}_{2}+\mathrm{H}_{2} \mathrm{O}$ plasma treated at $1 \times 10^{-2}$ mbar for $15 \mathrm{~min}$. The PP spectrum shows a broader and approximately 250 times stronger signal intensity due to the application of a charge compensation with the low-energy electron flood gun.

present, what confirms the possible hydrogen bound between the big $\mathrm{SO}_{3} \mathrm{H}$ group and the stabilizing $\mathrm{CN}$ functionalities. Unoxidized sulfur, $\mathrm{SO}$, and $\mathrm{SO}_{2}$ compounds are found on samples treated with higher ion energy (see Sec. III C).

\section{B. How many sulfur groups can be incorporated on the highly oriented pyrolytic graphite top surface?}

To estimate the surface coverage by the sulfur functionalities, the atomic percentage of each species bas been first calculated, assuming that the analysis depth corresponds to the electron mean-free path and that one $\mathrm{SO}_{3} \mathrm{H}$ molecule occupies the same space as three $\mathrm{C}$ atoms. With these approximations, one monolayer of $\mathrm{SO}_{3} \mathrm{H}$ at the HOPG surface induces sulfur XPS signal of $5 \%$ at an electron take-off angle of $90^{\circ}$. The maximal incorporation on HOPG and on GC always remains under 5\% (4\%-4.5\%). Taking into account that angle dependent measurements show that the sulfur is not only incorporated at the top surface, the coverage with sulfur functionalities is smaller than one monolayer, but at least half of a monolayer.

For the second estimation, we consider the sulfur functionalities as a homogeneous thin film on HOPG. With this approximation and for our instrument, the ratio of the intensities of the substrate $(s)$ and the film. $(f)$ is given by

$$
\frac{I_{f}}{I_{s}}=\frac{n_{f} s_{f}}{n_{s} s_{s}} \cdot\left(\frac{E_{f}}{E_{s}}\right)^{0.35} \exp \left(d / \lambda_{s}\right) \cdot\left(1-\exp \left(d / \lambda_{f}\right)\right)
$$




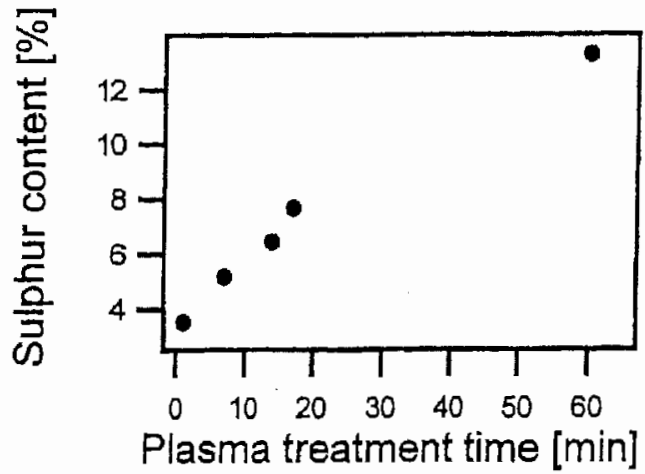

FIG. 5. XPS measured sulfur incorporation on $\mathrm{PP}$ as a function of $\mathrm{SO}_{2}$ $+\mathrm{H}_{2} \mathrm{O}$ plasma treatment time.

where $I_{f}$ is the intensity of the film, $I_{s}$ is the intensity of the substrate, $n$ is the density, $\sigma$ is the photoionization cross section, $E$ is the kinetic energy, $d$ is the film thickness, and $\lambda$ is mean-free path. One monolayer of $\mathrm{SO}_{3} \mathrm{H}$ on graphite corresponds to a ratio $I_{f} / I_{s}=0.5 \pm 0.05$. The $I_{f} / I_{s}$ ratio for our samples is comprised between 0.3 and 0.5 depending on the treatment conditions. Taking into account once again that a small part of the sulfur in incorporated into the bulk of the substrate, this estimation also predict a $\mathrm{SO}_{3} \mathrm{H}$ coverage of 0.5 to one monolayer.

\section{Dependence of the sulfur oxidation degree on the treatment conditions}

In comparison to other plasma treatments ${ }^{7}$ where the steady-state conditions are rapidly reached ( $<1 \mathrm{~min})$, the sulfur incorporation follows a slow process. Figure 5 presents the sulfur content of PP surfaces after a $\mathrm{SO}_{2}+\mathrm{H}_{2} \mathrm{O}$ plasma treatment as a function of the treatment time. The sulfur content seems to increase continuously with increasing treatment times. A similar but less pronounced behavior has been found for the HOPG surfaces. The deposited sulfur functionalities, which are big chemical groups (at least five atoms for $\mathrm{SO}_{3}$ and $\mathrm{N}$ ), are probably formed in homogeneous reactions such as:

$$
\mathrm{SO}_{2}+\mathrm{H}_{2} \mathrm{O} \rightarrow \mathrm{SO}_{2}^{*}+\mathrm{OH}^{*}+\mathrm{H} \rightarrow \mathrm{SO}_{3} \mathrm{H}+\mathrm{H} \text {. }
$$

The higher oxidation state $\left(\mathrm{SO}_{4} \mathrm{H}\right)$ is the result of the aforementioned homogeneous reaction and a direct oxidation of the substrate (heterogeneous reaction). The slow formation of such big molecules can be explained by the low probably of the homogeneous reaction and by the low content of nitrogen in the residual gas.

The occurrence of sulfur with various oxidation degrees depends on the treatment conditions. Figure 6 show the variation of the incorporated sulfur on HOPG as a function of the pressure and of the dc potential. The total amount of incorporated sulfur is about constant, even if the higher ion energies (low pressure or high dc potentials) seem to favor a greater incorporation. The oxygen and nitrogen contents have also been reported in Fig. 6(a), They are proportional to the sulfur content, as has been explained in Fig. 2, and the proportionality factor for $\mathrm{PP}$ is also of 1 for the nitrogen and of 4 for the oxygen. However, treatments at low pressures $\left(1 \times 10^{-4} \mathrm{mbar}\right)$ or with a dc. potential, favor the formation
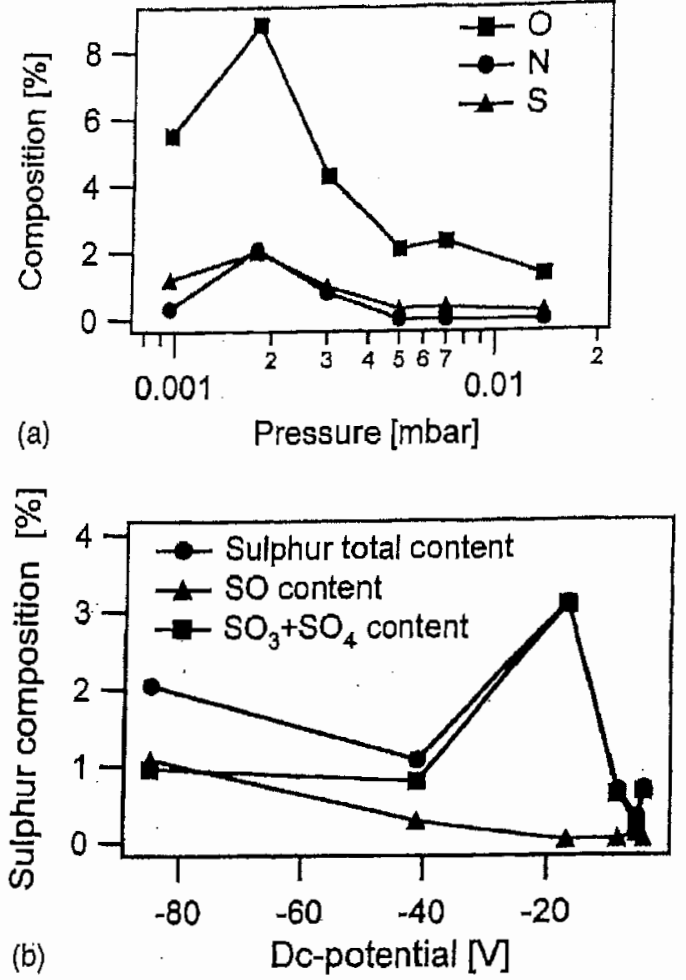

FIG. 6. Composition of $\mathrm{SO}_{2}+\mathrm{H}_{2} \mathrm{O}$ plasma treated HOPG surlaces for 5 min: (a) oxygen, nitrogen, and sulfuir percentage as a function of the pressure for floating potential and (b) total amount of sulfur divided into low oxidized and highly oxidized species as a function of de potential at 1 $\times 10^{-2}$ mbar.

of sulfur species with a lower degree of oxidation, as can be seen in Fig. 6(b). By decreasing the gas pressure or by increasing the dc potential, the ion energy is increased and, therefore, also the total energy deposited on the surface region. In these conditions, the plasma has greater etching properties and can destroy highly oxidized sulfur species at the substrate surface. Moreover, the lifetime of big molecules in the gaseous phase becomes shorter. This explains the formation of smaller new chemical species such as SO. For sufficiently high dc potential (high ion energy), the highly oxidized sulfur species almost disappear, and the SO molecules are incorporated into a thicker surface region. At low ion energies, $\mathrm{SO}_{2}$ plasma treatments were able to incorporate highly oxidized sulfur species, but the SO groups remain a majority (Fig. 7). Moreover, no hydrogen atoms, as well as too few oxygen atoms, are present in $\mathrm{SO}_{2}$ gas to form $\mathrm{SO}_{3} \mathrm{H}$ groups. To fill this gap, $\mathrm{H}_{2} \mathrm{O}$ vapor has been added in the gas flux. The addition of a low amount of $\mathrm{H}_{2} \mathrm{O}$ drastically increases the highly oxidized sulfur content of the surface to the detriment of $\mathrm{SO}$, as can be seen in Fig. 7. For an $\mathrm{H}_{2} \mathrm{O} / \mathrm{SO}_{2}$ ratio greater than one, the total amount of incorporated sulfur decreases rapidly. Similarly, the only impact of the addition of other gases such as $\mathrm{H}_{2}, \mathrm{O}_{2}$, or $\mathrm{N}_{2}$ is to diminish the total incorporation of sulfur and particularly of the highly oxidized sulfur compound.

The sulfur species incorporated by the plasma treatment, and particularly the SO molecules, are not stable and disappear progressively both on HOPG and on PP. For example, a decrease of $25 \%$ of the sulfur surface content is measured on HOPG $1 \mathrm{~h}$ in air or in a vacuum after the plasma treatment. 


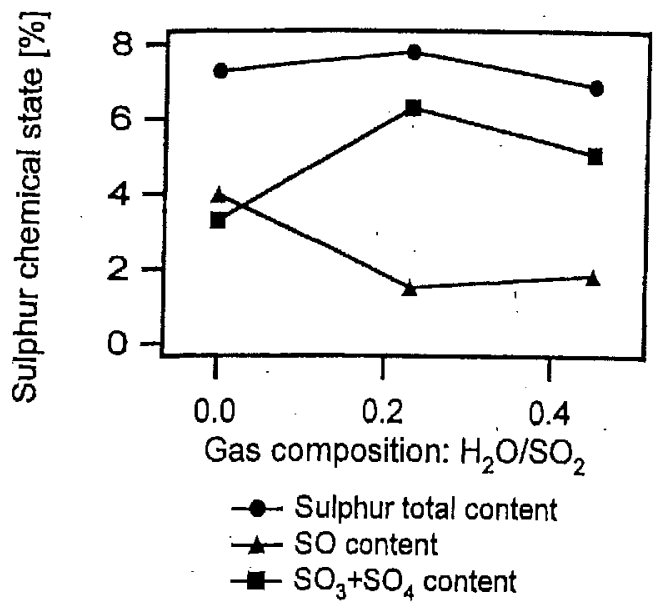

FIG. 7. Total amount of incorporated sulfur into $\mathrm{SO}_{2}+\mathrm{H}_{2} \mathrm{O}$ plasma treated HOPG strfaces, as well as low oxidized and highly oxidized species percentages as a function of the amount of water vapor into the plasma gas.

On the same material, $40 \%$ of the sulfur as well as $25 \%$ of the oxygen have disappeared $14 \mathrm{~h}$ after the plasma treatment. The nitrogen content remains however stable. Lin et al. ${ }^{8}$ have also measured a $25 \%$ decrease of the sulfur content in polyethylene after several days of storage. This sulfur disappearance from the surface region is probably due to a desorption of low molecular species containing sulfur.

\section{Functionalization of various polymers with sulfur species}

All the treatments on polymer surfaces have been performed at $1 \times 10^{-2}$ mbar during $15 \mathrm{~min}$ and with a gases mixture of $3.5 \mathrm{sccm} \mathrm{SO}$ and $0.6 \mathrm{sccm} \mathrm{H}_{2} \mathrm{O}$. The maximal incorporation of sulfur species for PP, TPU, PC, and PHB is given in Table II and is about $10 \%$ for all the measured polymers. The sulfur incorporation is then far greater on polymers than on graphite, where the maximal incorporation never exceeds $4.5 \%$. It is however clear that new chemical species are more easily introduced in polymers that are less crystalline substrates, because their densities are weaker and their surface cohesion smaller.

The maximal incorporation always corresponds to treatments at floating potential, that is at low ion energy, and is composed mostly of highly oxidized sulfur (Table II). The introduction of only highly oxidized sulfur on the surface is possible for PP, TPU, and PHB. BEs between 169.1 and $170.0 \mathrm{eV}$ are then found for all polymers, which correspond to $\mathrm{SO}_{3}(\mathrm{H})$ or $\mathrm{SO}_{4}(\mathrm{H})$ functionalities. SO and highly oxidized species are always simultaneously incorporated in PC.

TABLE II. The sulfur maximal content, the percentage of highly oxidized sulfur in the sulfur peak, the $B E$ of this highly oxidized peak, and the ratio between the nitrogen incorporated and the highly oxidized sulfur are given for several polymers

\begin{tabular}{lcccc}
\hline & S maximal content (\%) & h.o.S (\%) & h.o.S BE (eV) & N/h.o.S ratio \\
\hline PP & 13.3 & 79 & 169.59 & 1 \\
TPU & 9.5 & 100 & 169.09 & 1 \\
PC & 12.9 & 73 & 170.04 & 0.8 \\
PHB & 10.0 & 69 & 169.10 & 0.6 \\
\hline
\end{tabular}
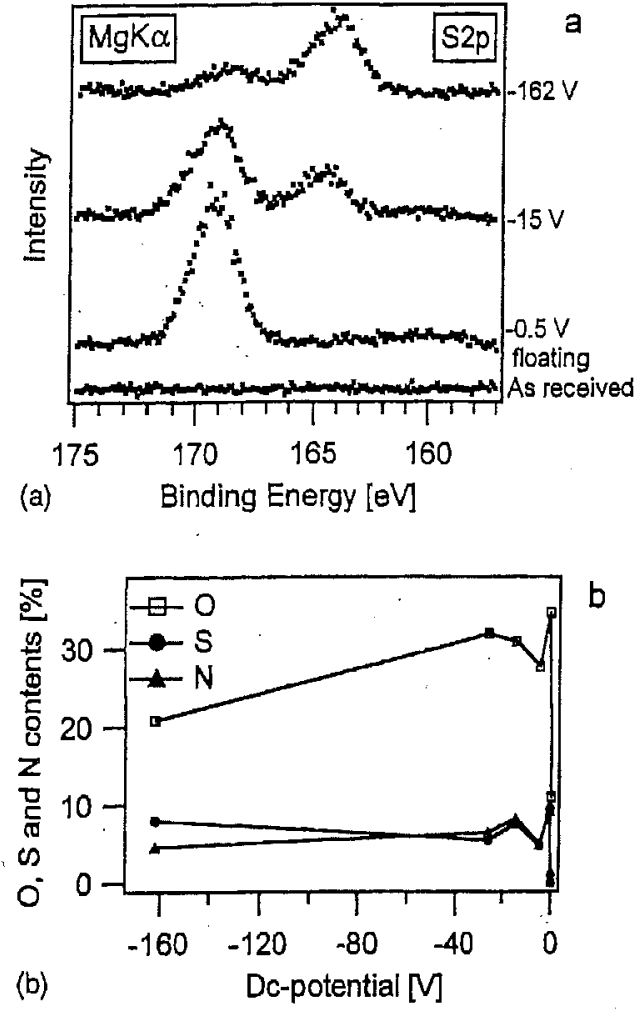

FIG. 8. XPS mensurements of $\mathrm{SO}_{2}+\mathrm{H}_{2} \mathrm{O}$ plasma treated PU surface at 1 $\times 10^{-2}$ mbar for 5 min and several dc potentials (a) S $2 p$ spectra and (b) oxygen, sulfur, and nitrogen surface contents as a function of the de potentials.

The dependence of the sulfur incorporation as a function of the different treatment conditions is absolutely similar to the one on HOPG. For higher ion energy, i.e., for greater dc potentials or for lower pressures, less sulfur can be incorporated $(3 \%-8 \%)$ and the $\mathrm{SO} / \mathrm{SO}_{3}$ ratio (XPS S $2 p$ peak at $164.0 \mathrm{eV}$ ) increases. This behavior can be visualized in Fig. 8 , where the S 2p XPS spectra and the oxygen, nitrogen, and sulfur contents of plasma treated TPU are reported as a function of the dc potential. Highly oxidized sulfur is found at low potential, with an optimum at floating potential. The amount of highly oxidized sulfur decreases with greater negative dc potentials and the one of SO increases. In summary, the maximal incorporation, the nature of the sulfur functionalities, and the dependence of the plasma parameters are identical for all investigated polymers. The fact that the functionalization of polymers with sulfur is independent of the type of the polymer implies a homogeneous formation of the highly oxidized sulfur species.

For PP and TPU, the ratio between the incorporated nitrogen and the highly oxidized sulfur content (N/h.o.S) is always about one (see Fig. 8). For TPU, greater nitrogen incorporation takes sometimes place (N/h.o.S $>1)$ when a dc potential is applied. For PC, on the contrary, the N/h.o.S ratio is always about 0.8 . Finally, the need to incorporate simultaneously nitrogen with highly oxidized sulfur on PHB is far smaller; the N/h.o.S ratio decreases to 0.2 .

\section{E. Topography of the $\mathrm{SO}_{2}$ plasma treated polypropylene and glassy carbon surfaces}

The nontreated biaxially oriented PP surface [Fig. 9(a)] shows a smooth texture with fiberlike structures probably 

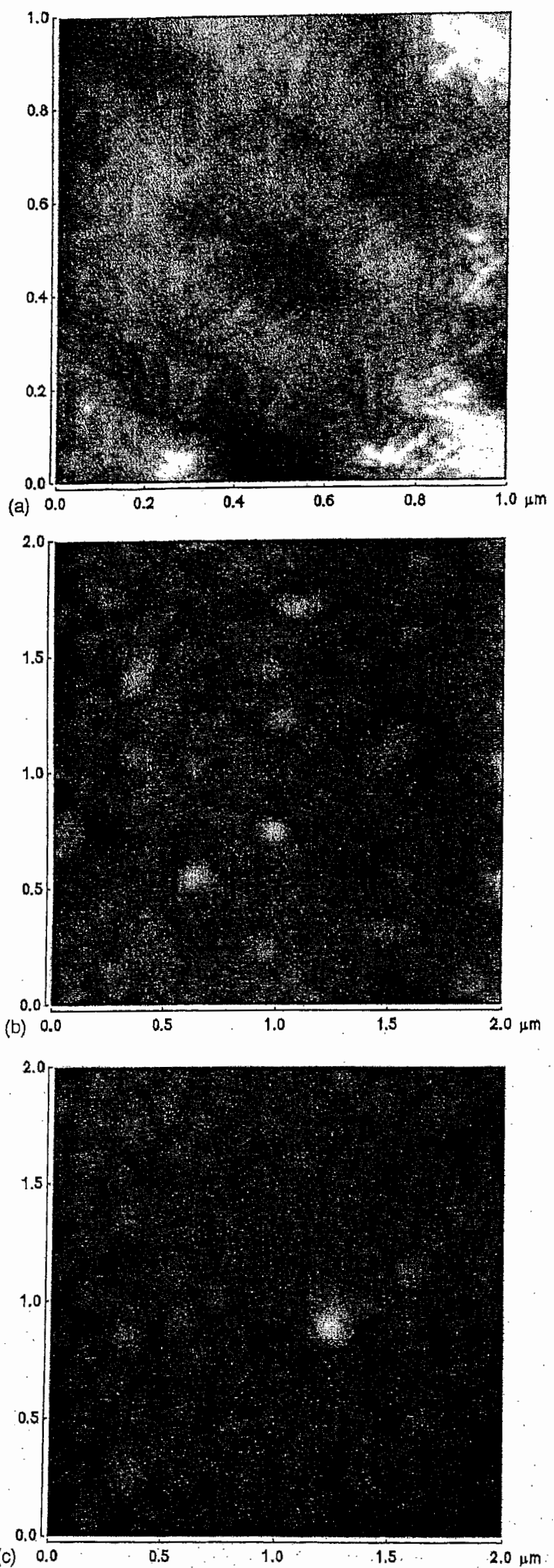

FIG. 9. AFM picturess of PP surfaces (a) as-received, (b) treated by $\mathrm{SO}_{2}$ plasma for $15 \mathrm{~min}$ at floating potential $\mathrm{V}=-2.5 \mathrm{~V}$, and (c) treated by $\mathrm{SO}_{2}$ plasma during 15 min with an additional dc bias of $-200 \mathrm{~V}$. The vertical scales are, respectively, $40 \mathrm{~nm}, 65 \mathrm{~nm}$, and $300 \mathrm{~nm}$.
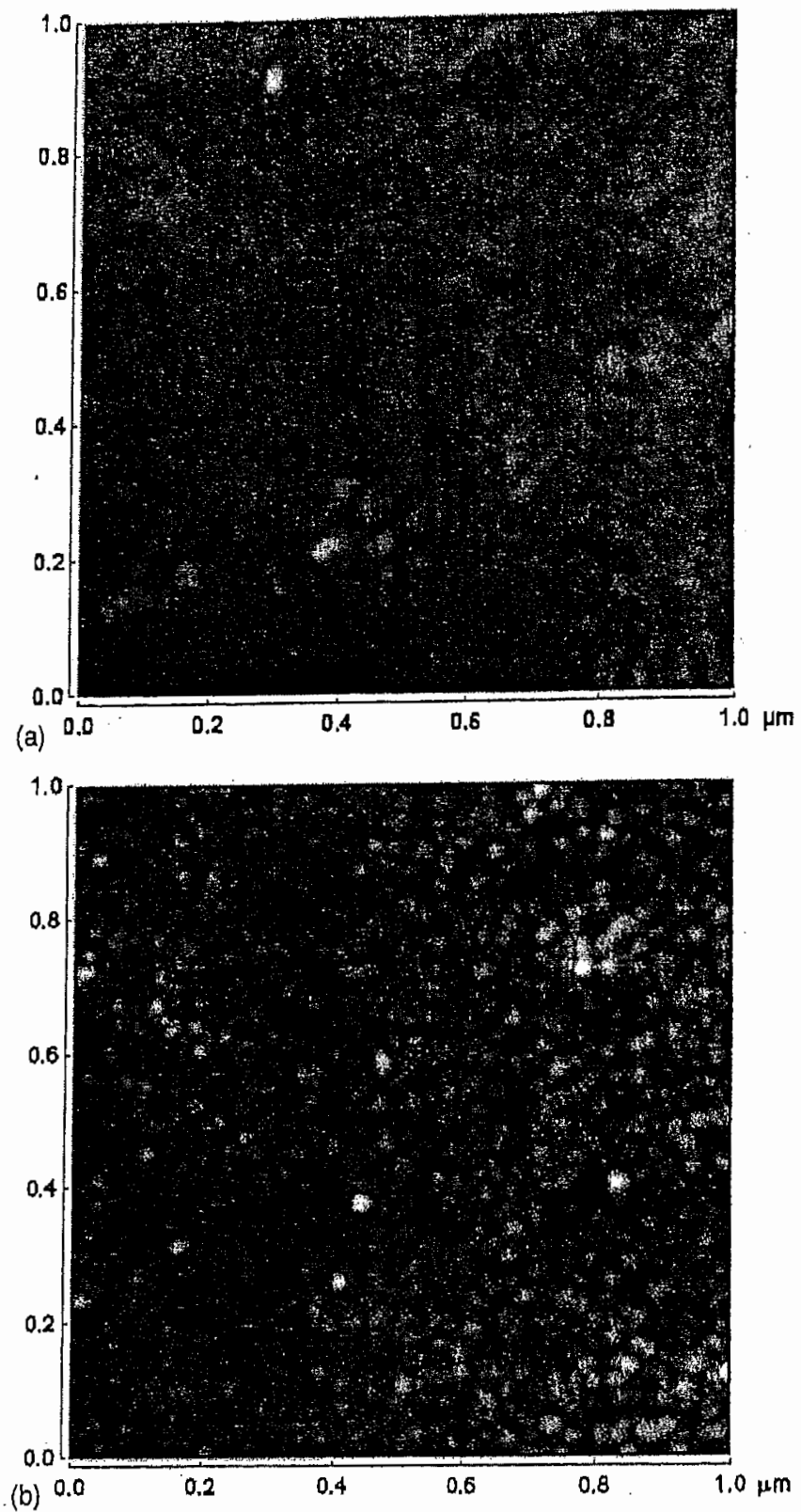

FIG. 10, AFM pictures of GC surfaces (a) as-received and (b) $\mathrm{SO}_{2}$ plasmia treated during $15 \mathrm{~min}$ at floating potential. The vertical scales are $17 \mathrm{~nm}$ and $14 \mathrm{~nm}$, respectively.

induced by the biaxially stretching of the film. Reactive gas plasma treatments, such as $\mathrm{N}_{2}, \mathrm{O}_{2}, \mathrm{H}_{2}$ or air, leave these structures almost in fact due to the stretching of the film, and generate some holes and protrusions on the surface. ${ }^{9,10}$ Only one of the reactive gases tested, $\mathrm{SO}_{2}$, is able to deeply modify the PP surface topography. Figure 9 shows the PP surface treated with $\mathrm{SO}_{2}$ plasma for $15 \mathrm{~min}$ [Fig. 9(b) at a floating potential and [Fig. $9(\mathrm{c})$ at $-200 \mathrm{~V}$. The sample treated at a floating potential $(-2.5 \mathrm{~V})$ has lost its original structure and presents big aggregates that are $80-150 \mathrm{~nm}$ wide and $15-25 \mathrm{~nm}$ higher than the mean surface height. Some smaller and lower aggregates are also clearly visible in the background. The roughness of the surface is, however, not changed $(\mathrm{rms}=9-10 \mathrm{~nm})$. The sample treated with higher ion energy [Fig. 9(c)] is uniformly composed of a dense packing of great aggregates that are $120-150 \mathrm{~nm}$ wide and $100 \mathrm{~nm}$ high. Its surface roughness has been greatly in- 
creased by the $\mathrm{SO}_{2}$ plasma treatment and is of $38 \mathrm{~nm} . \mathrm{SO}_{2}$ plasma is well known to be a hard etching treatment. Its peculiar abrasive properties are probably the reason to obtain such topographical modifications on PP.

As-received GC (Fig. 10) has a granular smooth surface with a rms of $2.1 \mathrm{~nm} . \mathrm{SO}_{2}$ plasma treatments induce an etching of the GC topography that evidences the granular structure without increasing the surface roughness (rms $=1.7 \mathrm{~nm}$ ). The grains have a diameter between 10 and 40 $\mathrm{nm}$. Due to the convolution of the AFM tip on these structures, the real size of the grains can be estimated to range between 2 and $20 \mathrm{~nm}$. This is approximately the size of the graphitelike ribbons. ${ }^{11}$ Brown et al. ${ }^{12}$ claim that the plasma etching left only the amorphous part of the GC at the surface. In this sense, the plasma reveals the pores between the graphitic phases. It is well known that the diamondlike amorphous carbon is less easily etched away than the graphite phases. However, our GC has a very high degree of crystallinity (97\%), so that measured grains have to correspond to the graphitic phases and not to the amorphous phase.

\section{CONCLUSION}

Unoxidized, low oxidized, and high oxidized sulfur species can be incorporated on graphitic and polymer surfaces by $\mathrm{SO}_{2}$ and $\mathrm{SO}_{2}+\mathrm{H}_{2} \mathrm{O}$ plasma treatments. The degree of oxidation depends on the energy of the ion of the plasma, which can be modified by either the pressure or the dc potential. The addition of water vapor facilitates the incorporation of $\mathrm{SO}_{3} \mathrm{H}$ and $\mathrm{SO}_{4} \mathrm{H}$, that can lead to protonic conductivity. The maximal incorporation on HOPG corresponds to more than one half of a monolayer of sulfur functionalities on the surface. The uptake of sulfur is much easier on polymers than on graphite. The behavior as a function of the plasma parameters is however completely similar for all measured substrates and the different degree of sulfur oxida- tion can be obtained on all samples. The incorporation of highly oxidized species always involves the presence of nitrogen at the surface, which is never found otherwise. We tentatively explain this phenomenon by a stabilizing effect of nitrogen on the big sulfur species via hydrogen bonding. The modification of topography during the plasma treatments can be closely linked to the etching property of $\mathrm{SO}_{2}$ plasma.

\section{ACKNOWLEDGMENTS}

The deep gratitude of the authors goes to Valérie Lalanne, Stéphane Berger, and Roland Lehmann, who partly did the XPS and AFM measurements, and to Zaharias Veziridis and Güther Scherrer for providing the GC and the HOPG.

' J. J.'Pouch and S. A. Alterovitz, Plasma Properties, Deposition, and Etching (Trans Tech Publications, Switzerland, 1993).

${ }^{2}$ H. V. Boenig, Fundamentals of Plasma Chemistry and Technology (Landcaster, Pa., 1988)

${ }^{3}$ S. Nowak, P. Groening, O. M. Küttel, M. Collaud, and G. Dietler, J. Vac. Sci. Technol. A 10, 3419 (1992).

${ }^{4} \mathrm{G}$. Beamson and D. Briggs, High Resolution XPS of Organic Polymers (Wiley, New York, 1992).

${ }^{5}$ P. Groening, M. Collaud Coen, O. M. Küttel, and L. Schlapbach, Appl. Surf. Sci. 103, 79 (1996).

${ }^{6}$ K. Siegbahn, C. Nordling, A. Fahlman, R. Nordberg, K. Hamrin, J. Hedman, G. Johansson, T. Bergmark, S. Karlsson, I. Lindgren, and B. Lindberg, Nova Acta Regiae Soc. Sci. Ups. 20, 1 (1968).

${ }^{7}$ M. Collaud, S. Nowak, O. M. Küttel, P. Groening, and L. Schlapbach, Appl. Surf. Sci. 72, 19 (1993)

${ }^{8}$ J. C. Lin, T. M. Ko, and S. L. Cooper, J. Colloid Interface Sci. 164, 99 (1994).

${ }^{9}$ M. Collaud Coen, G. Dietler, S. Kasas, and P. Groening, Appl. Surf. Sci. 103, 27 (1996).

${ }^{10} \mathrm{M}$. Collaud Coen and P. Groening, Proceeding of the Fifth International Conference on Adhesion and Surface Analysis (Loughbourough University Publisher, Loughbourough, UK, 1998), p. 1.

"M. S. Dresselhaus, G. Dresselhaus, and P. C. Eklund, Science of Fullerenes and Carbon Nanotubes (Academic, San Diego, 1996).

${ }^{12}$ N. M. D. Brown, N. Cui, and A. McKinley, Appl. Surf. Sci. 133, 157 (1998), 